This is an electronic reprint of the original article. This reprint may differ from the original in pagination and typographic detail.

\author{
Author(s): Müller-Gatermann, C.; Dewald, A.; Fransen, C.; Braunroth, T.; Jolie, J.; Litzinger, J.; \\ Regis, J. M.; von Spee, F.; Warr, N.; Zell, K. O.; Grahn, Tuomas; Greenlees, Paul; \\ Hauschild, Karl; Jakobsson, Ulrika; Julin, Rauno; Juutinen, Sakari; Ketelhut, Steffen; \\ Nieminen, Päivi; Nyman, Markus; Peura, Pauli; Rahkila, Panu; Ruotsalainen, Panu; \\ Sandzelius, Mikael; Sarén, Jan; Scholey, Catherine; Sorri, Juha; Stolze, Sanna; Uusitalo, \\ 1..han. natl.... n \\ Title: Low-lying electromagnetic transition strengths in $180 \mathrm{Pt}$
}

Year: $\quad 2018$

Version:

Please cite the original version:

Müller-Gatermann, C., Dewald, A., Fransen, C., Braunroth, T., Jolie, J., Litzinger, J., Regis, J. M., von Spee, F., Warr, N., Zell, K. O., Grahn, T., Greenlees, P., Hauschild, K., Jakobsson, U., Julin, R., Juutinen, S., Ketelhut, S., Nieminen, P., Nyman, M., . . . Petkov, P. (2018). Low-lying electromagnetic transition strengths in 180 Pt. Physical Review C, 97(2), Article 024336. https://doi.org/10.1103/PhysRevC.97.024336

All material supplied via JYX is protected by copyright and other intellectual property rights, and duplication or sale of all or part of any of the repository collections is not permitted, except that material may be duplicated by you for your research use or educational purposes in electronic or print form. You must obtain permission for any other use. Electronic or print copies may not be offered, whether for sale or otherwise to anyone who is not an authorised user. 


\title{
Low-lying electromagnetic transition strengths in ${ }^{180} \mathrm{Pt}$
}

\author{
C. Müller-Gatermann, ${ }^{1,}{ }^{*}$ A. Dewald,${ }^{1}$ C. Fransen, ${ }^{1}$ T. Braunroth, ${ }^{1}$ J. Jolie, ${ }^{1}$ J. Litzinger, ${ }^{1}$ J. M. Régis, ${ }^{1}$ F. von Spee, ${ }^{1}$ N. Warr, \\ K. O. Zell, ${ }^{1}$ T. Grahn, ${ }^{2}$ P. T. Greenlees, ${ }^{2}$ K. Hauschild, ${ }^{2}$ U. Jakobsson, ${ }^{2}$ R. Julin, ${ }^{2}$ S. Juutinen, ${ }^{2}$ S. Ketelhut, ${ }^{2}$ P. Nieminen, ${ }^{2}$ \\ M. Nyman, ${ }^{2}$ P. Peura, ${ }^{2}$ P. Rahkila, ${ }^{2}$ P. Ruotsalainen, ${ }^{2}$ M. Sandzelius, ${ }^{2}$ J. Sarén, ${ }^{2}$ C. Scholey, ${ }^{2}$ J. Sorri, ${ }^{2}$ \\ S. Stolze, ${ }^{2}$ J. Uusitalo, ${ }^{2}$ and P. Petkov ${ }^{3}$ \\ ${ }^{1}$ Institut für Kernphysik der Universität zu Köln, Zülpicher Strasse 77, D-50937 Köln, Germany \\ ${ }^{2}$ University of Jyvaskyla, Department of Physics, P.O. Box 35, FI-40014, University of Jyvaskyla, Finland \\ 3 “Horia Hulubei” National Institute for Physics and Nuclear Engineering, R-76900, Bucharest-Magurele, Romania
}

(Received 26 October 2017; published 27 February 2018)

\begin{abstract}
Lifetime measurements have been performed using the ${ }^{98} \mathrm{Mo}\left({ }^{86} \mathrm{Kr}, 4 n\right){ }^{180} \mathrm{Pt}$ reaction at a beam energy of $380 \mathrm{MeV}$, and the recoil distance Doppler-shift method. In a second experiment the ${ }^{168} \mathrm{Yb}\left({ }^{16} \mathrm{O}, 4 n\right){ }^{180} \mathrm{Pt}$ reaction at a beam energy of $88 \mathrm{MeV}$ using the Ge-gated $\gamma-\gamma$ fast timing technique was used to determine lifetimes. Lifetimes of the four lowest yrast states of ${ }^{180} \mathrm{Pt}$ have been determined. The experimental data are compared to calculations within the framework of the interacting boson model and the general collective model. Both models predict a deformed ground state and are consistent with all the remaining experimental results.
\end{abstract}

DOI: 10.1103/PhysRevC.97.024336

\section{INTRODUCTION}

Even-even nuclei close to shell closures are a subject of numerous experimental and theoretical studies [1,2]. One of the challenges is to understand the shape evolution in the chains of nuclei when one type of nucleon approaches magicity. In the case of the platinum isotopic chain the proton number is close to the shell closure at $Z=82$. Phenomena such as shape coexistence [2] and shape transitions [3] are observed in this region near the neutron $N=104$ midshell. The mean lifetime $\tau$ of excited states is an experimental observable from which one can extract model-independent transition probabilities that can be compared to theoretical approaches. In this context the model-dependent quadrupole deformation parameter $\beta$ can also be derived.

A simple correlation for the description of the $B\left(E 2,0_{1}^{+} \rightarrow\right.$ $2_{1}^{+}$) values in even-even nuclei was proposed by Casten [4]. It is a function of the valence neutrons $N_{\nu}$ and valence protons $N_{\pi}$, and the leading term is the product $N_{\nu} N_{\pi}$. According to this relation, a rapid change in nuclear shape is expected compared to the neighboring isotopic chains around the proton shell closure at $Z=82$ for valence neutron numbers around midshell. For the case of ${ }_{78} \mathrm{Pt}$ the neighboring isotopic chains are ${ }_{76} \mathrm{Os}$ and ${ }_{80} \mathrm{Hg}$. The ${ }^{176,178,180}$ Os isotopes are known candidates [5] for the critical point symmetry X(5) [6] proposed by Iachello. On the other hand the shapes of the neutron-deficient $\mathrm{Hg}$ isotopes [7] are determined by the excitation of intruder states, which cause a significant prolate deformation [8] coexisting with oblate ground states.

The purpose of the present work was to deduce $E 2$ transition strengths via lifetime measurements in the ground-state band of ${ }^{180} \mathrm{Pt}$ in order to extend the set of experimental

*cmgater@ikp.uni-koeln.de observables and clarify the situation between contradicting previous lifetime experiments $[9,10]$ for the $4_{1}^{+}$state. Furthermore, the spectroscopic properties like the level scheme as well as the transition probabilities are compared to theoretical models to characterize the nuclei in more detail. Especially if one needs multiparticle-multihole excitations to understand the observables in the isotopic chain of platinum is a long-lasting question [11]. In this work the approach of McCutchan et al. [11] was used again to reproduce the spectroscopic properties without intruder states, including the transition strengths which were not known at this time or were incorrect. For this purpose two collective models were used: The interacting boson model (IBM-1) [12] within the extended consistent $Q$-formalism (ECQF) [13] and the general collective model (GCM) [14].

\section{EXPERIMENTAL DETAILS}

\section{A. Recoil distance Doppler-shift experiment}

To populate excited states in ${ }^{180} \mathrm{Pt}$, the ${ }^{98} \mathrm{Mo}\left({ }^{86} \mathrm{Kr}, 4 n\right)$ reaction was used. The beam with an energy of $380 \mathrm{MeV}$ was provided by the K-130 cyclotron of the University of Jyväskylä. The target consisted of $0.9 \mathrm{mg} / \mathrm{cm}^{2}$ isotopically enriched ${ }^{98}$ Mo. After a fusion-evaporation reaction, the resulting nuclei were stopped in a $15 \mathrm{mg} / \mathrm{cm}^{2}$ gold foil, after a flight in vacuum with a mean velocity of about $4.4 \%$ of the velocity of light. Both the target and the stopper foils were mounted in the DPUNS plunger [15] (differential plunger for unbound nuclear states), which follows the design of the Cologne coincidence plunger [16]. The target-to-stopper distance was monitored by measuring the capacitance, and beam-induced drifts were compensated with a piezoelectric device. The plunger device was coupled to the JUROGAM II Ge-detector array, which consisted of two rings of EUROGAM (European gamma-ray microscope) clover detectors [17] around $90^{\circ}$ and two rings 


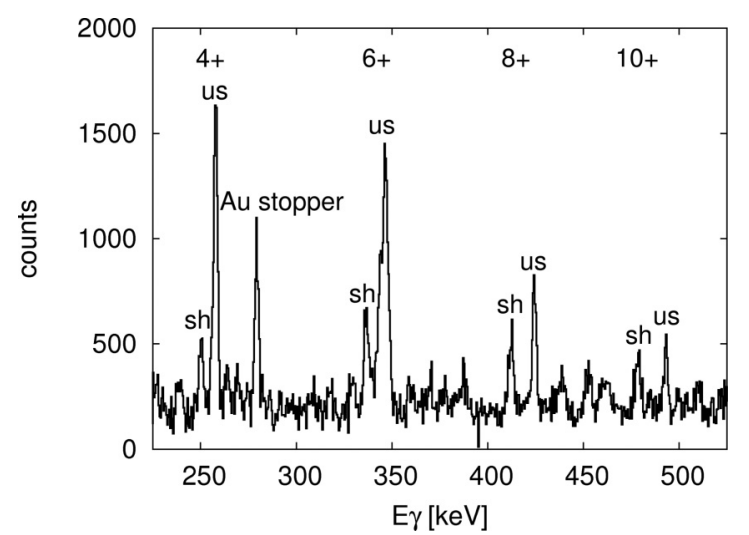

FIG. 1. $\gamma$-ray energy spectrum measured with the detectors in the ring at $133^{\circ}$ (10 detectors). This is the sum over all distances with a gate on both components of the $2_{1}^{+} \rightarrow 0_{1}^{+}$transition (including the clover detectors around $90^{\circ}$ ). Despite one random coincidence with the strong Coulomb excitation of ${ }^{197} \mathrm{Au}$ the spectrum is very clean. The initial states with their depopulating shifted and unshifted components are marked with "sh" and "us", respectively.

with tapered EUROGAM Phase I [18] or GASP (gamma-ray spectrometer) type [19] germanium detectors at $133^{\circ}$ and $157^{\circ}$ with respect to the beam direction. All detectors were Compton suppressed. Data were taken for 18 target-to-stopper distances ranging from electrical contact to $12 \mathrm{~mm}$. The cross section was only around $0.5 \mathrm{mb}$ resulting in a low count rate for the reaction to ${ }^{180} \mathrm{Pt}$, where only the JUROGAM II detectors at $133^{\circ}$ and $157^{\circ}$ can be used for the recoil distance Doppler-shift (RDDS) experiment. Nevertheless, it was possible to analyze the data by gating on the Doppler-shifted component of each direct feeding transition of the respective level of interest. Using the differential decay curve method (DDCM) [20], one is independent of any unobserved side-feeding effects. The normalization between the distances was done via Coulomb excitation of ${ }^{197} \mathrm{Au}$ in the gold stopper. The target was changed once during the experiment. Thus a constant correction factor had to be introduced to the two groups of distances with different targets.

Figure 1 shows the total statistics of the experiment in the ring at $133^{\circ}$ consisting of 10 detectors. It is summed up over all distances with a gate on both components of the $2_{1}^{+} \rightarrow 0_{1}^{+}$ transition. This shows not only the quality of the spectra but also the low $\gamma$-ray intensities resulting from the low reaction cross section to ${ }^{180} \mathrm{Pt}$.

\section{B. Electronic timing experiment}

Since it was not possible to deduce the lifetime of the rather long-lived $2_{1}^{+}$state within the RDDS experiment, a second experiment on ${ }^{180} \mathrm{Pt}$ was performed at the FN tandem accelerator of the University of Cologne using the fast electronic timing technique. This method is sensitive to lifetimes starting around $10 \mathrm{ps}$ up to several ns, whereas the RDDS method can be used to determine lifetimes between hundreds of fs up to hundreds of ps. The Horus (high efficiency observatory for $\gamma$-ray unique spectroscopy) cube spectrometer [21] was
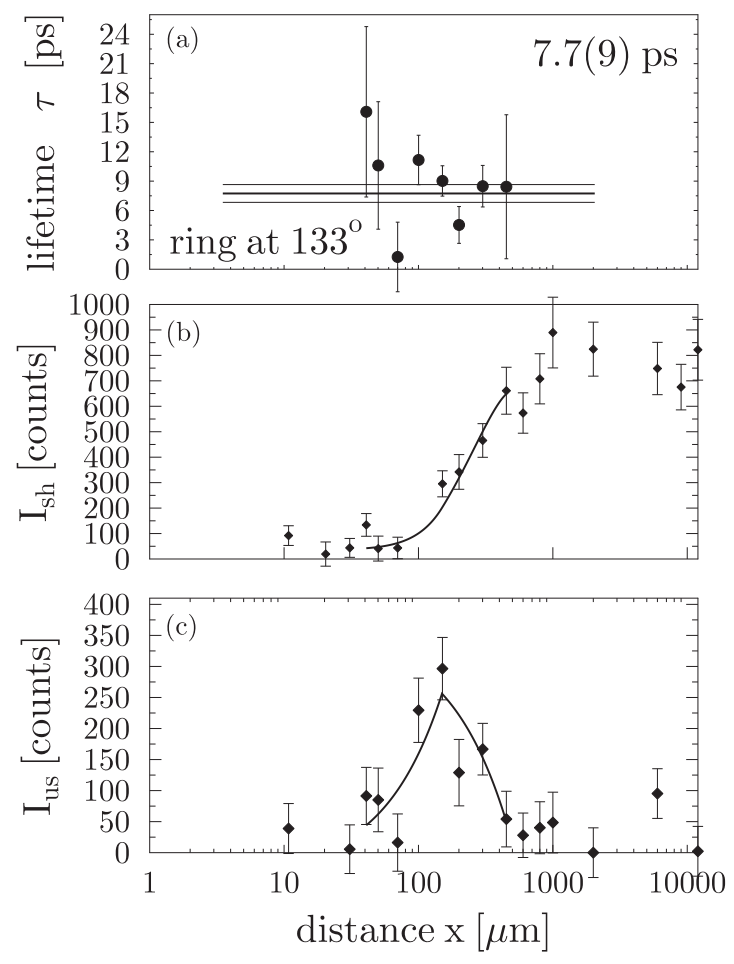

FIG. 2. $\tau$-curve (a) of the $6_{1}^{+}$state in ${ }^{180} \mathrm{Pt}$ using the detector ring at $133^{\circ}$ with a gate on the feeding $8_{1}^{+}$state in all backward angle detectors. In addition, the intensities of the shifted (b) and unshifted (c) components are shown.

equipped with eight $\mathrm{LaBr}_{3}(\mathrm{Ce})$ scintillation detectors $(\mathrm{LaBr})$, six of which were equipped with anti-Compton shields, two detectors in a $\mathrm{Pb}$ shield were fixed inside the spectrometer as additional detectors and in intermediate positions. The remaining eight positions were used with standard single-crystal highpurity germanium (HPGe) detectors. The ${ }^{168} \mathrm{Yb}\left({ }^{16} \mathrm{O}, 4 n\right){ }^{180} \mathrm{Pt}$ reaction at a beam energy of $88 \mathrm{MeV}$ was chosen for the triple $\gamma$ coincidence measurement with a gate applied to the HPGe detectors to clean the $\mathrm{LaBr}$ spectrum from other reaction channels. The target was $1 \mathrm{mg} / \mathrm{cm}^{2}{ }^{168} \mathrm{Yb}$ enriched to $35.2 \%$ on a $2 \mathrm{mg} / \mathrm{cm}^{2}$ Au backing.

\section{LIFETIME ANALYSIS}

\section{A. DDCM}

At each distance $x$, the lifetime is calculated according to the formalism of the DDCM in "coincidence mode" presented in detail in Ref. [20]. The lifetime is obtained from

$$
\tau(x)=\frac{I_{\mathrm{us}}(x)}{\frac{d}{d x} I_{\mathrm{sh}}(x)} \frac{1}{v},
$$

where $x$ is the target to stopper distance, $I_{\mathrm{us}}$ and $I_{\mathrm{sh}}$ are the areas of the unshifted and shifted peaks of the transition of interest, respectively, and $v$ is the mean velocity of the recoiling nuclei. Equation (1) yields a set of lifetime values (the $\tau$ curve), one for each distance $x$, which should be independent of $x$. Since $\gamma \gamma$ coincidences were measured with a direct gate on the Doppler-shifted component of a feeding 
TABLE I. Experimental values of the lifetimes using different methods. The adopted values are marked with *. In the theoretical calculations a lifetime of 2.2(3) ps for the $8_{1}^{+}$was employed, since no other result for the lifetime existed.

\begin{tabular}{lcc}
\hline \hline State & $E_{\gamma}(\mathrm{keV})$ & $\tau(\mathrm{ps})$ \\
\hline $2_{1}^{+}$ & 153.2 & $420(20)^{\mathrm{a} *}$ \\
& & $420(30)^{\mathrm{b}}$ \\
& & $540(50)^{\mathrm{d}}$ \\
$4_{1}^{+}$ & 257.6 & $37(2)^{\mathrm{c} *}$ \\
& & $36(6)^{\mathrm{b}}$ \\
& & $75(14)^{\mathrm{d}}$ \\
& & $33(4)^{\mathrm{e}}$ \\
$6_{1}^{+}$ & 346.5 & $53(5)^{\mathrm{f}}$ \\
& & $7.7(9)^{\mathrm{c} *}$ \\
& & $8.8(10)^{\mathrm{g}}$ \\
$8_{1}^{+}$ & $13(3)^{\mathrm{f}}$ \\
& 424.3 & $2.2(3)^{\mathrm{c}}$ \\
& & $3.1(1)^{\mathrm{g}} *$ \\
\hline \hline
\end{tabular}

a This work using the convolution method.

${ }^{\mathrm{b}}$ This work using the GCD method.

${ }^{\mathrm{c}}$ This work using the RDDS method.

de Voigt et al. [9].

eWilliams et al. [10].

${ }^{\mathrm{f}}$ Chen et al. [24].

${ }^{\mathrm{g}}$ von Spee [25] using the RDDS method.

transition, no feeding has to be taken into account. Only a normalization between the distances was performed using the $\gamma$-ray lines from the Coulomb excitation of ${ }^{197} \mathrm{Au}$. Since the target was changed once during the experiment, a constant factor of 0.65 was introduced to correct the normalization for different targets. The application of Eq. (1) for the lifetime determination of the $6_{1}^{+}$state in ${ }^{180} \mathrm{Pt}$ is shown in Fig. 2 . The lifetimes using this analysis are $\tau\left(4_{1}^{+}\right)=37(2) \mathrm{ps}, \tau\left(6_{1}^{+}\right)=$ 7.7(9) ps, and $\tau\left(8_{1}^{+}\right)=2.2(3) \mathrm{ps}$ and are summarized together with the lifetimes obtained in other experiments in Table I. The

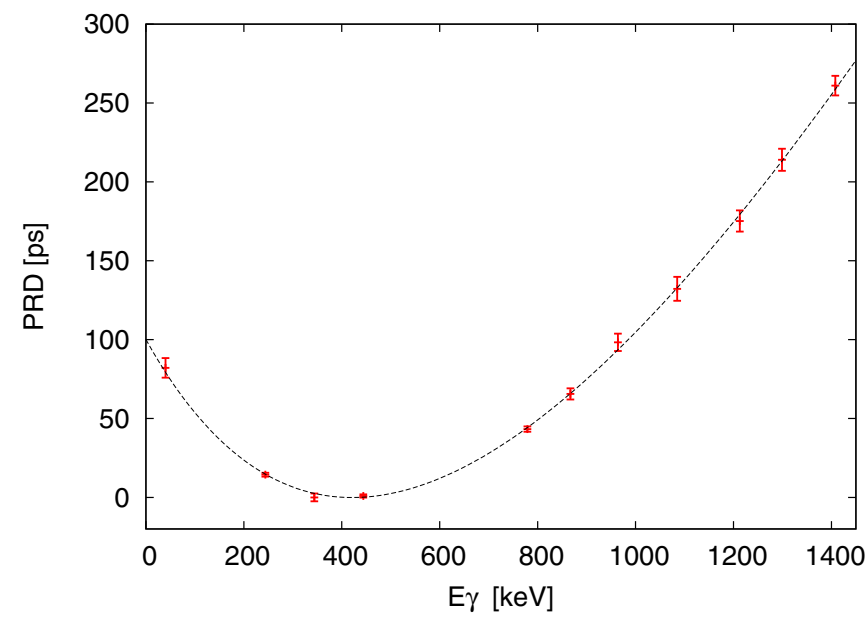

FIG. 3. PRD curve of the electronics using a ${ }^{152} \mathrm{Eu}$ source.

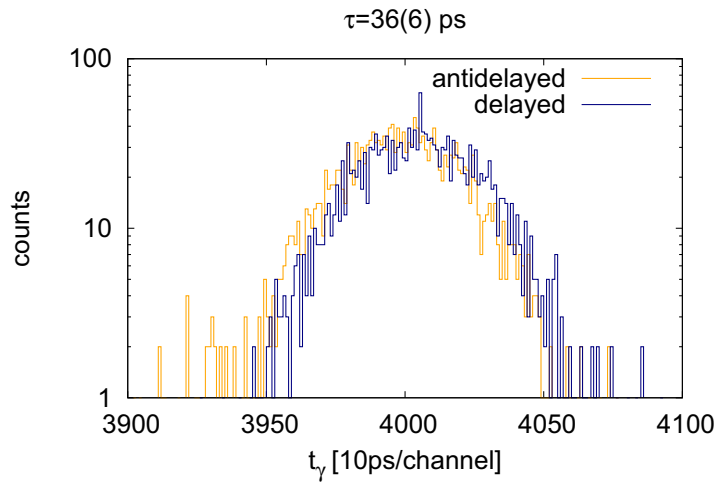

FIG. 4. Time-difference spectra of the $4_{1}^{+}$state. The difference of the centroids between the delayed and antidelayed spectra corresponds, after correction for the PRD, to twice the lifetime of the state.

$10^{+}$state in ${ }^{180} \mathrm{Pt}$ could not be analyzed due to contamination of a $\gamma$-ray line from ${ }^{182} \mathrm{Pt}$ with almost the same energy and the gate would have been in the tail of an intense ${ }^{197} \mathrm{Au} \gamma$ ray in addition to the decreasing statistics for higher lying states.

\section{B. Electronic timing}

By applying two energy gates on the $\mathrm{LaBr}$ detectors a feeder-decay cascade corresponding to a given state of interest can be selected. For every combination of $\mathrm{LaBr}$ detectors the setup provides two independent time difference spectra, the delayed and the antidelayed, measured as the time difference between a start and a stop detector. The delayed spectrum is produced when the feeding transition provides the start signal and the decay transition the stop signal. The antidelayed spectrum is incremented when the decay transition provides the start signal and the feeding transition the stop signal. To clean up the spectrum, an additional gate on a coincident $\gamma$-ray transition observed with a HPGe detector was applied, so triple coincidences were used for the lifetime analysis. Two lifetimes using two different methods were extracted, namely, the generalized centroid difference (GCD) [22] method for the $4_{1}^{+}$state and both the convolution method and the GCD method

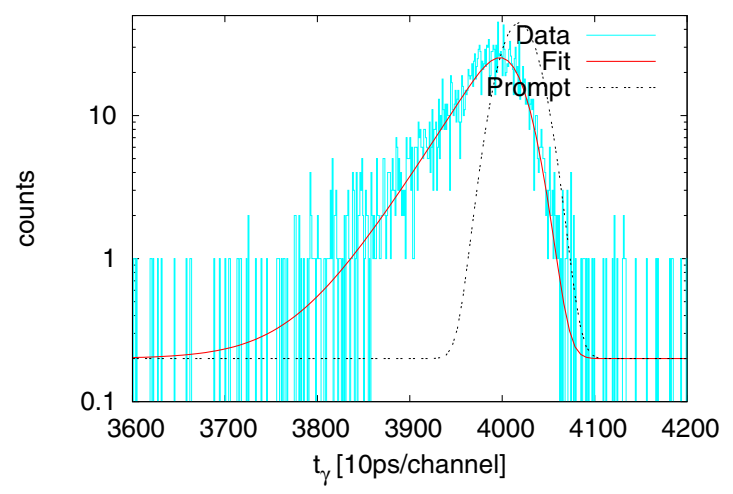

FIG. 5. Fit of the convolution of a prompt peak with the lifetime of the $2_{1}^{+}$state. Also the corresponding prompt peak (dotted) is plotted to show the low statistics in the tail. 


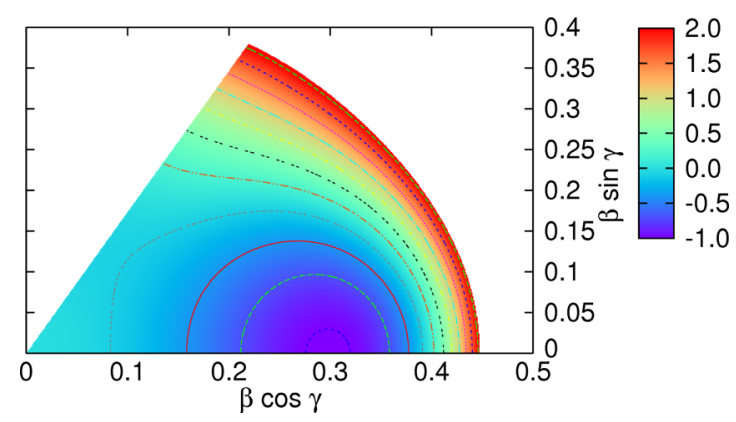

FIG. 6. Potential energy surface extracted from the GCM calculation.

for the $2_{1}^{+}$state. Details of the GCD method are presented in Ref. [22]. Since the displacement of the centroids of the delayed and antidelayed time distributions is equal to (twice) the mean lifetime of the state $\tau$ and an energy-dependent $\gamma-\gamma$ time walk of the system, known as the prompt response difference (PRD) [23], a PRD calibration with a ${ }^{152} \mathrm{Eu}$ source was determined and is shown in Fig. 3. The function to describe the form of the PRD is

$$
\operatorname{PRD}\left(E_{\gamma}\right)=\frac{a}{\sqrt{E_{\gamma}+b}}+c E_{\gamma}+d .
$$

The analysis of the lifetime of the $4_{1}^{+}$state is then straightforward since there is no background below the peak in the LaBr spectra. The time difference spectra are shown in Fig. 4. The result is in agreement with the lifetime using DDCM. Regarding the $2_{1}^{+}$state, there is still some background below the peak at $153 \mathrm{keV}$. One can correct for the timing behavior of the background events originating from Compton-scattered photons of higher energy with the formula [22]

$$
\Delta C_{\mathrm{FEP}}=\Delta C+\frac{\Delta C-\Delta C_{\mathrm{BG}}}{\Pi} .
$$

Here $\Delta C$ is a centroid difference, where BG and FEP stand for background and full energy peak, respectively, and $\Pi$ is the peak-to-background ratio. In fact the centroid difference for background events is the mean value of the combinations for background in the feeder (decay) coincident with an event in the decay (feeder) respectively. The result of 420(30) ps for the lifetime is reasonable, but due to the large peak-to-background ratio a second method was applied, the so-called convolution

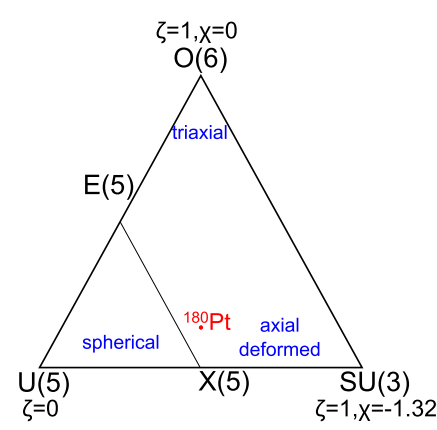

FIG. 7. Placement of ${ }^{180} \mathrm{Pt}$ in the Casten triangle. method. Here one fits a complete convolution of a prompt peak and a decay function using the lifetime as a fit parameter to the time spectra, given by the function

$$
F(t)=\frac{N_{0}}{\tau} e^{-\frac{t-t_{0}}{\tau}}\left[1-\operatorname{erf}\left(\frac{\sigma}{\sqrt{2} \tau}-\frac{t-t_{0}}{\sqrt{2} \sigma}\right)\right]
$$

(erf is the error function). For longer lifetimes, it is possible to restrict the fit to the tail of the time distribution in order to avoid the prompt peak. In this case, the lifetime is too short for the slope method as the statistics away from the prompt peak are too weak, resulting in a large error. The fit of the convolution is shown in Fig. 5, resulting in a lifetime of the $2_{1}^{+}$state of 420(20) ps and supporting the result of the GCD method. The deconvoluted prompt peak has the same width as prompt events in this energy regime, which is an indicator for a correct convolution, and it shows how many counts would remain for a slope fit. Using this method no correction of background events is taken into account, but the background events should correspond mostly to Compton-scattered $\gamma$ rays of higher lying transitions with shorter lifetimes. Under this assumption these counts are in the timing spectrum close to the prompt peak and do not affect the measured lifetime significantly. In fact the lifetime information is in the tail and the centroid difference between the expected Compton events and a prompt peak is less than $10 \mathrm{ps}$. This worst case scenario would most probably correspond to a Compton-scattered $\gamma$ ray of the decaying $6_{1}^{+}$ state, because the reaction channel is selected with the HPGe gate on the $8_{1}^{+}$decay.

\section{DISCUSSION OF RESULTS}

The lifetime of the first excited state in ${ }^{180} \mathrm{Pt}$ was remeasured and the value of de Voigt et al. [9] is longer than the value obtained in this work. This holds also for the $4_{1}^{+}$lifetime, where the data shown here are in good agreement with the lifetime of Williams et al. [10]. Especially it has to be noted that two independent methods within two experiments were used and the derived values agree with each other. At the time the experiment was performed the lifetimes of the $6_{1}^{+}$ and $8_{1}^{+}$states were unknown, but recently Chen et al. [24] also published lifetimes for these states. All their results for lifetimes of the lowest yrast states are significantly longer. The results in this work were then again confirmed by another RDDS experiment on ${ }^{180} \mathrm{Pt}$ aiming for the commissioning of the GALILEO plunger [26] using the ${ }^{154} \mathrm{Sm}\left({ }^{32} \mathrm{~S}, 6 n\right)$ reaction. This measurement was sensitive to the lifetimes of the $6_{1}^{+}$and $8_{1}^{+}$states (also shown in Table I) [25]. For the $8_{1}^{+}$state the GALILEO plunger commissioning run has a lot more statistics than the previous experiment, therefore this lifetime is given as an adopted value which is within the errorbars consistent with Chen et al. The error of the RDDS experiment is purely statistical and may be underestimated. Regarding the $6_{1}^{+}$state the lifetime result is confirmed again. The fact that the results for the lifetimes of the $4_{1}^{+}$and $6_{1}^{+}$states of Chen et al. are both longer hints at a systematic error of these data. 


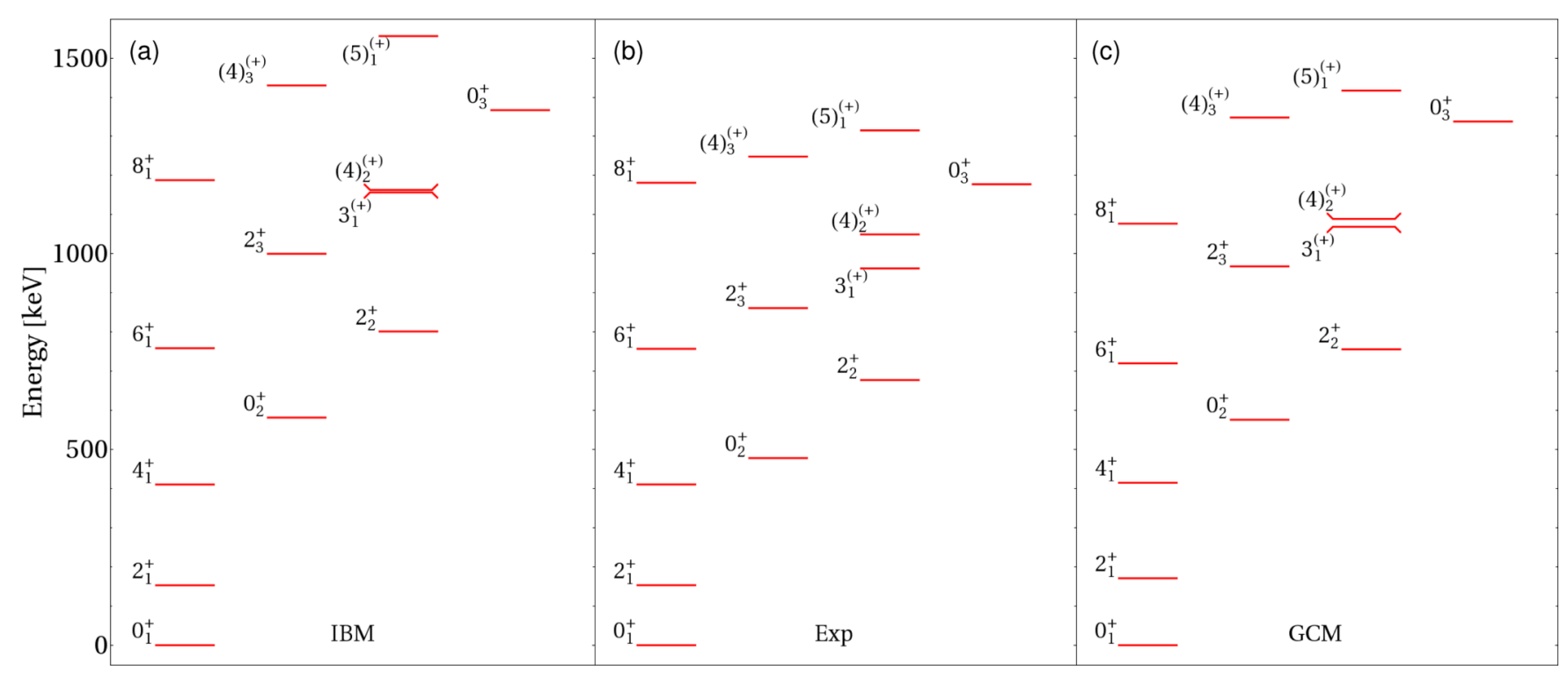

FIG. 8. Comparison of the IBM calculation (a), the experimental values (b), and the GCM calculation (c). Shown are the level energies for the ground state, $\beta$ and $\gamma$ bands, and the third $0^{+}$state. The $B(E 2)$ values are summarized in Table I.

\section{INTERPRETATION}

Two collective models were used to reproduce the level energies and $B(E 2)$ values of ${ }^{180} \mathrm{Pt}$. The first one is the IBM-1 [12] with the Hamiltonian in the extended consistent $Q$ formalism (ECQF) [13]

$$
\begin{aligned}
H(\zeta, \chi) & =c\left[(1-\zeta) \hat{n}_{d}-\frac{\zeta}{4 N_{B}} \hat{Q}^{\chi} \hat{Q}^{\chi}\right] \\
\hat{n}_{d} & =d^{\dagger} \tilde{d}, \quad \hat{Q}^{\chi}=\left[s^{\dagger} \tilde{d}+d^{\dagger} s\right]^{(2)}+\chi\left[d^{\dagger} \tilde{d}\right]^{(2)}, \\
\hat{T}(E 2) & =e_{B} \hat{Q}^{\chi},
\end{aligned}
$$

with $N_{B}$ being the boson number, $\hat{n}_{d}$ the $d$-boson number operator, $\hat{Q}^{\chi}$ the quadrupole operator, $s^{\dagger}, s, d^{\dagger}$, and $\tilde{d}$ the creation and annihilation operators for $s$ and $d$ bosons, $c$ a scaling parameter for the energies, $\hat{T}(E 2)$ the $E 2$ transition operator, $e_{B}$ the effective boson charge, and $\zeta, \chi$ the fitting parameters which can directly be used for classification within the Casten triangle [27]. $\zeta=0.57, \chi=-1.00, c=1.25$, and $e_{B}=0.18 e \mathrm{~b}$ were deduced for ${ }^{180} \mathrm{Pt}$ to reproduce the level scheme and $B(E 2)$ values. The uncertainty of these parameters corresponds to \pm 1 in the last digit given.

The second model used is the general collective model [14], which approximates the Bohr-Hamiltonian with the sum of kinetic energy $\hat{T}$ and collective potential $V(\beta, \gamma)$ expanded in a series of the quadrupole variables $\alpha_{2 \mu}$. The kinetic energy is

$$
\hat{T}=\frac{1}{2 B_{2}}[\hat{\pi} \times \hat{\pi}]^{[0]}+\frac{P_{3}}{3}\left\{\left[[\hat{\pi} \times \alpha]^{[2]} \times \hat{\pi}\right]^{[0]}\right\},
$$

where $\{\ldots\}$ means the sum over all even permutations of $\alpha$ and the conjugated momenta $\hat{\pi}$, while $B_{2}$ is the common mass parameter and $P_{3}$ accounts for the deformation-dependent inertial functions. The potential energy is

$$
\begin{aligned}
V(\beta, \gamma)= & C_{2} \frac{1}{\sqrt{5}} \beta^{2}-C_{3} \sqrt{\frac{2}{35}} \beta^{3} \cos 3 \gamma \\
& +C_{4} \frac{1}{5} \beta^{4}-C_{5} \sqrt{\frac{2}{175}} \beta^{5} \cos 3 \gamma \\
& +D_{6} \frac{1}{5 \sqrt{5}} \beta^{6}+C_{6} \frac{2}{35} \beta^{6} \cos ^{2} 3 \gamma,
\end{aligned}
$$

with the two intrinsic vibrational variables $\beta$ and $\gamma$, which are related to the quadrupole variables via the polar transformation $\alpha_{20}^{\text {intr }}=\beta \cos \gamma$ and $\alpha_{22}^{\text {intr }}=\beta \sin \gamma / \sqrt{2}$. Using the fit parameters $C_{2}=-24.98, C_{3}=195.60, C_{4}=312.60, C_{5}=$ $-2135.80, C_{6}=-613.70$, and $D_{6}=3051.20$ one can obtain directly the potential energy surface (PES) shown in Fig. 6. For completeness the mass parameters are $B_{2}=61.14$ and $P_{3}=0.0594$. One can summarize for both models that all level energies in the ground-state band are reproduced very well, in addition to the corresponding $B(E 2)$ values within the error bars. Also the $\beta$ and $\gamma$ bands together with the third $0^{+}$state are reproduced in the correct order with most relative transition strengths from known branching ratios [28] within the error bars. Given the parameters $\zeta$ and $\chi$ from the IBM- 1 calculation one can locate ${ }^{180} \mathrm{Pt}$ in the space spanned by them: the Casten triangle (Fig. 7). In addition to the three limits $U(5), S U(3)$, and $\mathrm{O}(6)$, which can be geometrically interpreted as a spherical vibrator, a prolate rotor, and a $\gamma$-soft rotor, the location of the critical point symmetries $\mathrm{E}(5)$ [29] and X(5) [6] are marked. One sees that ${ }^{180} \mathrm{Pt}$ is, as expected, close to the $\mathrm{X}(5)$ symmetry, but on the axially deformed rotor side. It has to be emphasized that in contrast to the $\mathrm{Hg}$ nuclei no mixing with proton $2 \mathrm{p}-2 \mathrm{~h}$ intruder excitation was introduced or needed to reproduce the spectroscopic properties with a high quality. García-Ramos et al. [30] published a series of model calculations for the platinum isotopic chain using these intruders and compared it 
to the simpler ECQ calculations by McCutchan et al. [11]. The values given in there are consistent with the parameters of the ECQ Hamiltonian used in this work. This means the parameters found to describe ${ }^{180} \mathrm{Pt}$ in a detailed way are consistent with parameters used to describe a larger series of nuclei. Also the PES from the GCM calculation shows a prolate deformation with some $\gamma$ softness for the ground state. The experimental and theoretical values from the models for the level energies and transition strengths are summarized in Fig. 8 and Table II.

In Fig. 9 experimental results of transition quadrupole moments $Q_{t}$ for the $N=102$ isotones are shown. Also given are the expectations for rotor, vibrator, and X(5)-like nuclei with increasing spin. It has been shown that multiparticle-hole excitations cause a large deformation in the neutron-deficient lead and mercury isotopes [1]. This effect is also observed in the yrast band of ${ }^{182} \mathrm{Hg}$. One can see an abrupt increase in $Q_{t}$ values of yrast transitions above the $2_{1}^{+}$state. Whereas ${ }^{180} \mathrm{Pt}$, ${ }^{178} \mathrm{Os}$, and ${ }^{176} \mathrm{~W}$ show a rather constant behavior. Observed variations are small and can be explained by different collective structures: For ${ }^{180} \mathrm{Pt}, Q_{t}$ values are constant and follow the rotor expectations; the same holds for ${ }^{176} \mathrm{~W}$ (unfortunately here

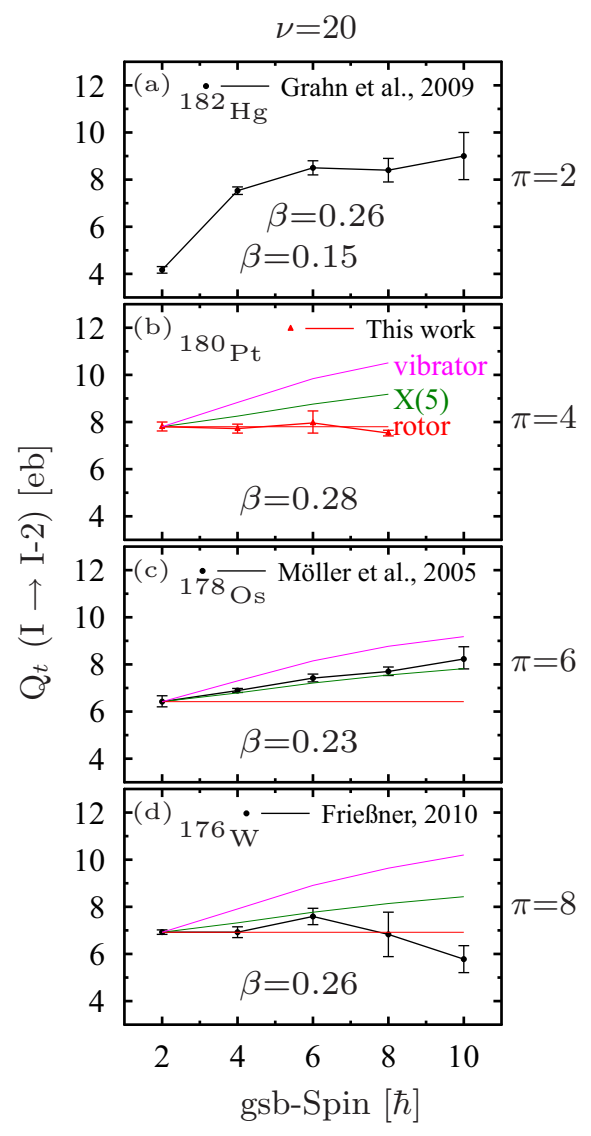

FIG. 9. Transition quadrupole moments for the $N=102$ isotone around Pt. $\pi$ and $v$ are the valence proton and neutron number, respectively. Experimental values taken from $[7,32,33]$. The indicated deformations are calculated from the lowest quadrupole moment neglecting variations described by the collective models; just for the case of ${ }^{182} \mathrm{Hg}$ the near constant deformation for the excited states is given as well.
TABLE II. Derived absolute $B(E 2)$ values from the experiments for the yrast band transitions in $e^{2} \mathrm{~b}^{2}$ as well as relative $B(E 2)$ values from known branching ratios [28] for the states in the $\beta$ and $\gamma$ bands and the third $0^{+}$state. For each state the level energy is given in $\mathrm{keV}$. The experimental data of ${ }^{180} \mathrm{Pt}$ are compared to the predictions of the IBM and GCM calculations.

\begin{tabular}{|c|c|c|c|c|c|c|c|}
\hline \multirow[t]{2}{*}{$J_{i}^{\pi}$} & \multirow[t]{2}{*}{$J_{f}^{\pi}$} & \multicolumn{3}{|c|}{$E_{\text {level }}[\mathrm{keV}]$} & \multicolumn{3}{|c|}{$B(E 2)\left[e^{2} \mathrm{~b}^{2}\right]$} \\
\hline & & IBM & Expt. & GCM & IBM & Expt. & GCM \\
\hline \multirow[t]{2}{*}{$2_{1}^{+}$} & & 153.1 & 153.3 & 171 & & & \\
\hline & $\rightarrow 0_{1}^{+}$ & & & & 1.13 & $1.20(6)$ & 1.09 \\
\hline \multirow[t]{2}{*}{$4_{1}^{+}$} & & 410.6 & 410.7 & 415 & & & \\
\hline & $\rightarrow 2_{1}^{+}$ & & & & 1.78 & $1.70(8)$ & 1.89 \\
\hline \multirow[t]{2}{*}{$6_{1}^{+}$} & & 758.8 & 757.1 & 720 & & & \\
\hline & $\rightarrow 4_{1}^{+}$ & & & & 2.1 & $1.9(2)$ & 2.4 \\
\hline \multirow[t]{2}{*}{$8_{1}^{+}$} & & 1188.1 & 1181.5 & 1077 & & & \\
\hline & $\rightarrow 6_{1}^{+}$ & & & & 2.2 & $2.6(4)$ & 2.8 \\
\hline $0_{2}^{+}$ & & 581.4 & 478.1 & 576 & & & \\
\hline \multirow{6}{*}{$2_{3}^{+}$} & & 988.7 & 861.4 & 968 & & & \\
\hline & $\rightarrow 2_{2}^{+}$ & & & & 324.36 & $400(300)$ & 158.23 \\
\hline & $\rightarrow 0_{2}^{+}$ & & & & 100 & 100 & 100 \\
\hline & $\rightarrow 4_{1}^{+}$ & & & & 56.7 & $61(7)$ & 60.99 \\
\hline & $\rightarrow 2_{1}^{+}$ & & & & 3.18 & $5.3(7)$ & 1.35 \\
\hline & $\rightarrow 0_{1}^{+}$ & & & & 9.72 & $12.4(9)$ & 7.34 \\
\hline \multirow[t]{5}{*}{$(4)_{3}^{(+)}$} & & 1415.9 & 1248.2 & 1348 & & & \\
\hline & $\rightarrow 2_{3}^{+}$ & & & & 100 & 100 & 100 \\
\hline & $\rightarrow 2_{2}^{+}$ & & & & 1.24 & $4(?)$ & 1.43 \\
\hline & $\rightarrow 4_{1}^{+}$ & & & & 1.34 & $0.9(7)$ & 1.19 \\
\hline & $\rightarrow 2_{1}^{+}$ & & & & 1.11 & $0.6(1)$ & 1.83 \\
\hline \multirow[t]{5}{*}{$2_{2}^{+}$} & & 801.5 & 677.5 & 756 & & & \\
\hline & $\rightarrow 0_{2}^{+}$ & & & & 214.95 & $200(200)$ & 98.41 \\
\hline & $\rightarrow 4_{1}^{+}$ & & & & 40.33 & $30(20)$ & 22.5 \\
\hline & $\rightarrow 2_{1}^{+}$ & & & & 100 & 100 & 100 \\
\hline & $\rightarrow 0_{1}^{+}$ & & & & 4.41 & $10.0(8)$ & 9.38 \\
\hline \multirow[t]{4}{*}{$3_{1}^{(+)}$} & & 1156.9 & 962.7 & 1069 & & & \\
\hline & $\rightarrow 2_{2}^{+}$ & & & & 100 & 100 & 100 \\
\hline & $\rightarrow 4_{1}^{+}$ & & & & 18.29 & $15(2)$ & 20.61 \\
\hline & $\rightarrow 2_{1}^{+}$ & & & & 11.62 & $17.1(7)$ & 16.44 \\
\hline \multirow[t]{4}{*}{$(4)_{2}^{(+)}$} & & 1163.2 & 1049.3 & 1089 & & & \\
\hline & $\rightarrow 2_{2}^{+}$ & & & & 100 & 100 & 100 \\
\hline & $\rightarrow 4_{1}^{+}$ & & & & 17.90 & $45(6)$ & 31.84 \\
\hline & $\rightarrow 2_{1}^{+}$ & & & & 0.04 & $1.4(7)$ & 0.85 \\
\hline \multirow[t]{3}{*}{$(5)_{1}^{(+)}$} & & 1556.1 & 1315.2 & 1417 & & & \\
\hline & $\rightarrow 3_{1}^{(+)}$ & & & & 100 & 100 & 100 \\
\hline & $\rightarrow 4_{1}^{+}$ & & & & 3.15 & $4.7(4)$ & 8.02 \\
\hline \multirow[t]{3}{*}{$0_{3}^{+}$} & & 1367.0 & 1177.7 & 1338 & & & \\
\hline & $\rightarrow 2_{2}^{+}$ & & & & 100 & 100 & 100 \\
\hline & $\rightarrow 2_{1}^{+}$ & & & & 0.83 & $22(2)$ & 0.01 \\
\hline
\end{tabular}


the experimental errors are larger, especially for the $6_{1}^{+}, 8_{1}^{+}$ states). ${ }^{178} \mathrm{Os}$ follows very nicely the $\mathrm{X}(5)$ prediction. Looking to the absolute $Q_{t}\left(2_{1}^{+} \rightarrow 0_{1}^{+}\right)$values, the corresponding $\beta$ deformation is given also in Fig. 9. For ${ }^{182} \mathrm{Hg}$ the $\beta$ deformation of the excited states caused by the intruder configuration is given as well. The deformation decreases approaching the shell closure from ${ }^{176} \mathrm{~W}$ to ${ }^{182} \mathrm{Hg}$ with one exception: ${ }^{180} \mathrm{Pt}$. The deformation found for ${ }^{180} \mathrm{Pt}$ is comparable to the deformation of the excited states in ${ }^{182} \mathrm{Hg}$. This strongly suggests that the large deformation in ${ }^{180} \mathrm{Pt}$ is caused by the intruder configuration becoming the ground state. This is supported by García-Ramos et al. [31] with a mean-field approach as well as an IBM calculation with $2 \mathrm{p}-2 \mathrm{~h}$ configuration mixing. However, the authors also admit that the configuration mixing is somehow "hidden" and does not show up explicitly as it does in the isotopic chains of mercury or lead.

\section{CONCLUSION}

Four lifetimes in the yrast band of ${ }^{180} \mathrm{Pt}$ were measured using three experiments to determine yrast $B(E 2)$ values. IBM-1 and GCM calculations were performed to describe the nuclear structures. They indicate both an axially deformed shape with some $\gamma$ softness and reproduce the level scheme and the $B(E 2)$ values quite well. Except for the large $Q_{t}$ values we have no indication of multiparticle-hole excitations. This is different from the isotopic chain of mercury and others where such excitations are needed to explain the nuclear properties $[2,8]$. A second note can be made that ${ }^{180} \mathrm{Pt}$ is clearly not following the expected trend for transition quadrupole moments in X(5) nuclei. The rather large quadrupole deformation in the neighboring ${ }^{182} \mathrm{Pt}$ (seen by Gladnishki et al. [34]) indicates that this is a general effect, which points to a weakening of the shell closure at $Z=82$. In the future one should investigate also the transition quadrupole moments for the lighter ${ }^{176,178} \mathrm{Pt}$ isotopes and compare them to the calculations by García-Ramos et al. [31]. It is suggested that the weakening of the shell closure is apparent only down to ${ }^{178} \mathrm{Pt}$ and the deformation should decrease for the lower masses. So far the lifetime information on these nuclei is too sparse, but reachable with standard fusion-evaporation reactions and stable beams; they would serve as a perfect testing ground.

\section{ACKNOWLEDGMENTS}

This work was supported by the Deutsche Forschungsgemeinschaft (DFG) under Contracts No. DE 1516/3-1 and No. JO 391/16-1, by the EU 7th Framework Programme, "Integrating Activities - Transnational Access", Project No. 262010 (ENSAR), and by the Academy of Finland under the Finnish Centre of Excellence Programme (Nuclear and Accelerator Based Physics Programme at JYFL). The authors acknowledge the support of GAMMAPOOL for the loan of the JUROGAM II detectors.
[1] T. Grahn, A. Dewald, O. Möller, R. Julin, C. W. Beausang, S. Christen, I. G. Darby, S. Eeckhaudt, P. T. Greenlees, A. Görgen, K. Helariutta, J. Jolie, P. Jones, S. Juutinen, H. Kettunen, T. Kröll, R. Krücken, Y. Le Coz, M. Leino, A.-P. Leppänen, P. Maierbeck, D. A. Meyer, B. Melon, P. Nieminen, M. Nyman, R. D. Page, J. Pakarinen, P. Petkov, P. Rahkila, B. Saha, M. Sandzelius, J. Sarén, C. Scholey, J. Uusitalo, M. Bender, and P.-H. Heenen, Nucl. Phys. A 801, 83 (2008).

[2] K. Heyde and J. L. Wood, Rev. Mod. Phys. 83, 1467 (2011).

[3] P. Cejnar, J. Jolie, and R. F. Casten, Rev. Mod. Phys. 82, 2155 (2010).

[4] R. F. Casten, Nucl. Phys. A 443, 1 (1985).

[5] A. Dewald, O. Möller, B. Saha, K. Jessen, A. Fitzler, B. Melon, T. Pissulla, S. Heinze, J. Jolie, K. O. Zell, P. von Brentano, P. Petkov, S. Harissopulos, G. De Angelis, T. Martinez, D. R. Napoli, N. Marginean, M. Axiotis, C. Rusu, D. Tonev, A. Gadea, Y. H. Zhang, D. Bazzacco, S. Lunardi, C. A. Ur, R. Menegazzo, and E. Farnea, J. Phys. G: Nucl. Part. Phys. 31, S1427 (2005).

[6] F. Iachello, Phys. Rev. Lett. 87, 052502 (2001).

[7] T. Grahn, A. Petts, M. Scheck, P. A. Butler, A. Dewald, M. B. Gomez Hornillos, P. T. Greenlees, A. Görgen, K. Helariutta, J. Jolie, P. Jones, R. Julin, S. Juutinen, S. Ketelhut, R. Krücken, T. Kröll, M. Leino, J. Ljungvall, P. Maierbeck, B. Melon, M. Nyman, R. D. Page, T. Pissulla, P. Rahkila, J. Sarén, C. Scholey, A. Semchenkov, J. Sorri, J. Uusitalo, R. Wadsworth, and M. Zielinska, Phys. Rev. C 80, 014324 (2009).

[8] J. E. García-Ramos and K. Heyde, Phys. Rev. C 89, 014306 (2014).
[9] M. De Voigt, R. Kaczarowski, H. Riezebos, R. F. Noorman, J. Bacelar, M. Deleplanque, R. Diamond, F. Stephens, J. Sauvage, and B. Roussière, Nucl. Phys. A 507, 472 (1990).

[10] E. Williams, C. Plettner, E. A. McCutchan, H. Levine, N. V. Zamfir, R. B. Cakirli, R. F. Casten, H. Ai, C. W. Beausang, G. Gürdal, A. Heinz, J. Qian, D. A. Meyer, N. Pietralla, and V. Werner, Phys. Rev. C 74, 024302 (2006).

[11] E. A. McCutchan, R. F. Casten, and N. V. Zamfir, Phys. Rev. C 71, 061301(R) (2005).

[12] A. Arima and F. Iachello, Phys. Rev. Lett. 35, 1069 (1975).

[13] D. D. Warner and R. F. Casten, Phys. Rev. C 28, 1798 (1983).

[14] G. Gneuss and W. Greiner, Nucl. Phys. A 171, 449 (1971).

[15] M. J. Taylor, D. M. Cullen, A. J. Smith, A. McFarlane, V. Twist, G. A. Alharshan, M. G. Procter, T. Braunroth, A. Dewald, E. Ellinger, C. Fransen, P. A. Butler, M. Scheck, D. T. Joss, B. Saygi, C. G. McPeake, T. Grahn, P. T. Greenlees, U. Jakobsson, P. Jones, R. Julin, S. Juutinen, S. Ketelhut, M. Leino, P. Nieminen, J. Pakarinen, P. Peura, P. Rahkila, P. Ruotsalainen, M. Sandzelius, J. Sarén, C. Scholey, J. Sorri, S. Stolze, and J. Uusitalo, Nucl. Instrum. Methods A 707, 143 (2013).

[16] A. Dewald, O. Möller, and P. Petkov, Prog. Part. Nucl. Phys. 67, 786 (2012).

[17] G. Duchêne, F. A. Beck, P. J. Twin, G. de France, D. Curien, L. Han, C. W. Beausang, M. A. Bentley, P. J. Nolan, and J. Simpson, Nucl. Instrum. Methods A 432, 90 (1999).

[18] C. W. Beausang, S. A. Forbes, P. Fallon, P. J. Nolan, P. J. Twin, J. N. Mo, J. C. Lisle, M. A. Bentley, J. Simpson, F. A. Beck, D. Curien, G. de France, G. Duchêne, and D. Popescu, Nucl. Instrum. Methods A 313, 37 (1992). 
[19] C. R. Alvarez, Nucl. Phys. News 3, 10 (1993).

[20] A. Dewald, S. Harissopulos, and P. von Brentano, Z. Phys. A Atomic Nuclei 334, 163 (1989).

[21] A. Linnemann, Ph.D thesis, Universität zu Köln, 2006.

[22] J.-M. Régis, G. S. Simpson, A. Blanc, G. de France, M. Jentschel, U. Köster, P. Mutti, V. Paziy, N. Saed-Samii, T. Soldner, C. A. Ur, W. Urban, A. M. Bruce, F. Drouet, L. M. Fraile, S. Ilieva, J. Jolie, W. Korten, T. Kröll, S. Lalkovski, H. Mach, N. Mărginean, G. Pascovici, Z. Podolyak, P. H. Regan, O. J. Roberts, J. F. Smith, C. Townsley, A. Vancraeyenest, and N. Warr, Nucl. Instrum. Methods A 763, 210 (2014).

[23] J.-M. Régis, M. Rudigier, J. Jolie, A. Blazhev, C. Fransen, G. Pascovici, and N. Warr, Nucl. Instrum. Methods A 684, 36 (2012).

[24] Q. M. Chen, X. G. Wu, Y. S. Chen, C. B. Li, Z. C. Gao, G. S. Li, F. Q. Chen, C. Y. He, Y. Zheng, S. P. Hu, J. Zhong, Y. H. Wu, H. W. Li, and P. W. Luo, Phys. Rev. C 93, 044310 (2016).

[25] F. von Spee, Bachelor thesis, Universität zu Köln, 2016.
[26] A. Goasduff, C. Fransen, A. Dewald, S. Thiel, T. Braunroth, C. Müller-Gatermann, D. Bazzacco, P. Cocconi, A. Gozzelino, K. Hadyńska-Klȩk, G. Jaworski, P. R. John, D. Mengoni, F. Recchia, M. Siciliano, D. Testov, J. J. ValienteDobón, and F. von Spee, in LNL Annual Report 2015, INFN, Laboratori Nazionali di Legnaro, 2016, pp. 91-92 (unpublished).

[27] R. F. Casten, Nuclear Structure from a Simple Perspective, 2nd ed. (Oxford University, Oxford, 2000).

[28] E. A. McCutchan, Nucl. Data Sheets 126, 151 (2015).

[29] F. Iachello, Phys. Rev. Lett. 85, 3580 (2000).

[30] J. E. García-Ramos and K. Heyde, Nucl. Phys. A 825, 39 (2009).

[31] J.E. García-Ramos, K. Heyde, L. M. Robledo, and R. RodríguezGuzmán, Phys. Rev. C 89, 034313 (2014).

[32] O. Möller, Ph.D. thesis, Universität zu Köln, 2005.

[33] G. Frießner, Diploma thesis, Universität zu Köln, 2010.

[34] K. A. Gladnishki, P. Petkov, A. Dewald, C. Fransen, M. Hackstein, J. Jolie, T. Pissulla, W. Rother, and K. Zell, Nucl. Phys. A 877, 19 (2012). 